\title{
Topological defects after a quench in a Bénard-Marangoni convection system
}

\author{
S. Casado, W. González-Viñas, H. Mancini, and S. Boccaletti \\ Department of Physics and Applied Mathematics, Universidad de Navarra, Irunlarrea s/n, 31080 Pamplona, Spain
}

(Received 5 October 2000; revised manuscripts received 3 January 2001; published 24 April 2001)

\begin{abstract}
We report experimental evidence of the fact that, in a Bénard-Marangoni conduction-convection transition, the density of defects in the emerging structure scales as a power law in the quench time needed for the control parameter to ramp through the threshold. The obtained scaling exponents differ from the ones predicted and observed in the case in which the defects correspond to zeros in the amplitude of the global two-dimensional field.
\end{abstract}

DOI: 10.1103/PhysRevE.63.057301

PACS number(s): 47.54.+r, 47.20.Ky, 47.27.Te

Pattern formation in space extended systems is a subject that has received much attention in nonlinear science during the last decades [1]. One of the most important features of a pattern-forming system is the appearance of phase singularities or topological defects, associated, in general, with a growing pattern. If one considers fields extended in two space dimensions, defects are pointlike structures such that the circulation of the phase gradient is a multiple of $2 \pi$ on any path surrounding them (thus the name "phase singularities'").

Defect formation, statistics, and dynamics were largely investigated in the past, both theoretically and experimentally [2,3]. Moreover, their role in mediating turbulence in large aspect ratio hydrodynamical systems was investigated in fluid thermal convection [4,5], in surface waves [6], and in numerical and analytical treatments of partial differential equations [7].

At this stage, it should be remarked that a topological defect of a two-dimensional field may be realized in different ways. A first possibility is having a point where the amplitude of a complex field vanishes, thus inducing a singularity in the phase. This is the case of a dislocation in a pattern where the field has a zero value in its amplitude and a discontinuity in its phase [3]. However, in a hexagonal pattern, three coupled normal modes with generally different amplitudes concur in the formation of the global field. In this case, the most stable topological defect is realized in a point of space wherein only the amplitudes of two modes vanish at once. An example of this latter situation is the appearance of the so called penta-hepta structure in hexagonal fields [5].

Recently it was argued that defects played a role in phase transitions occurring in the universe cooling process after the Big Bang, yielding to the apparition of galaxies [8]. The argument may be summarized as follows. Consider a system in a state just below a symmetry-breaking transition. By approaching the bifurcation point with the control parameter, the correlation length tries to adapt adiabatically to its stationary value, but, due to the slowing down near the critical point, its change rate is limited by a maximum velocity in the propagation of perturbations in the system. At the point at which the correlation length can no longer follow the changes in the control parameter, it freezes, setting up the initial value after the breaking symmetry transition, and thus also the initial density of defects in the structure. Furthermore, it is expected that this process leads to a universal scaling law for the defect density in the appearing structure, which depends only upon the space dimension, topology, and dissipative character of the system [8].

In order to give a solid ground to the above argument, several experiments were performed in nonequilibrium phase transition systems, among others in superfluid helium [9] and liquid crystals [10]. More recently, another kind of experiment, where the breaking supercritical transition was considered between two stationary nonequilibrium states, was reported in a nonlinear optical system [11].

Both theoretical [8] and experimental [11] evidence of universal scaling laws for the defect density in second order phase transitions focused on the case of defects generated as zeros in the amplitude of the global field. The purpose of this paper is twofold. We first aim at verifying that a scaling law is present in symmetry-breaking phase transitions, leading to the appearance of defects which are not zeros of the global field. Second, we aim at comparing these results with results obtained previously, pointing out analogies and differences between the two cases. The most important difference is that in the former case the defects correspond to points where the system retains the amplitude of the state before the bifurcation, while in the latter case this does not occur.

Here we report experimental results for the defect density in a conduction-convection bifurcation of a BénardMarangoni system. The considered bifurcation is a symmetry-breaking one. The state below the transition point is a homogeneous conduction state, while the state above the transition is a hexagonal pattern. This bifurcation was reported [12] as a subcritical one. Due to the small value of the subcriticality $\left(\epsilon_{\alpha}\right.$ is of the order of $10^{-2}$ ), for all quench times the system is already frozen when the control parameter $\epsilon$ reaches a value $\epsilon_{\alpha}$. As a result, we argue that this subcriticality is not relevant to the expected behavior of the system in the reported time quenches. In our experiment, the defect creation takes place at characteristic times on the order of $1 \mathrm{~min}$, while the pattern evolution time scale is on the order of $1 \mathrm{~h}$, thus allowing one to cross over the threshold and observe defects over a sufficiently large time range.

The experiments were performed with two similar setups [see Fig. 1(a)]. The convective fluid consists in a layer of silicone oil confined in a cylindric container (of polioximetilene and polyamide, both having thermal conduc- 

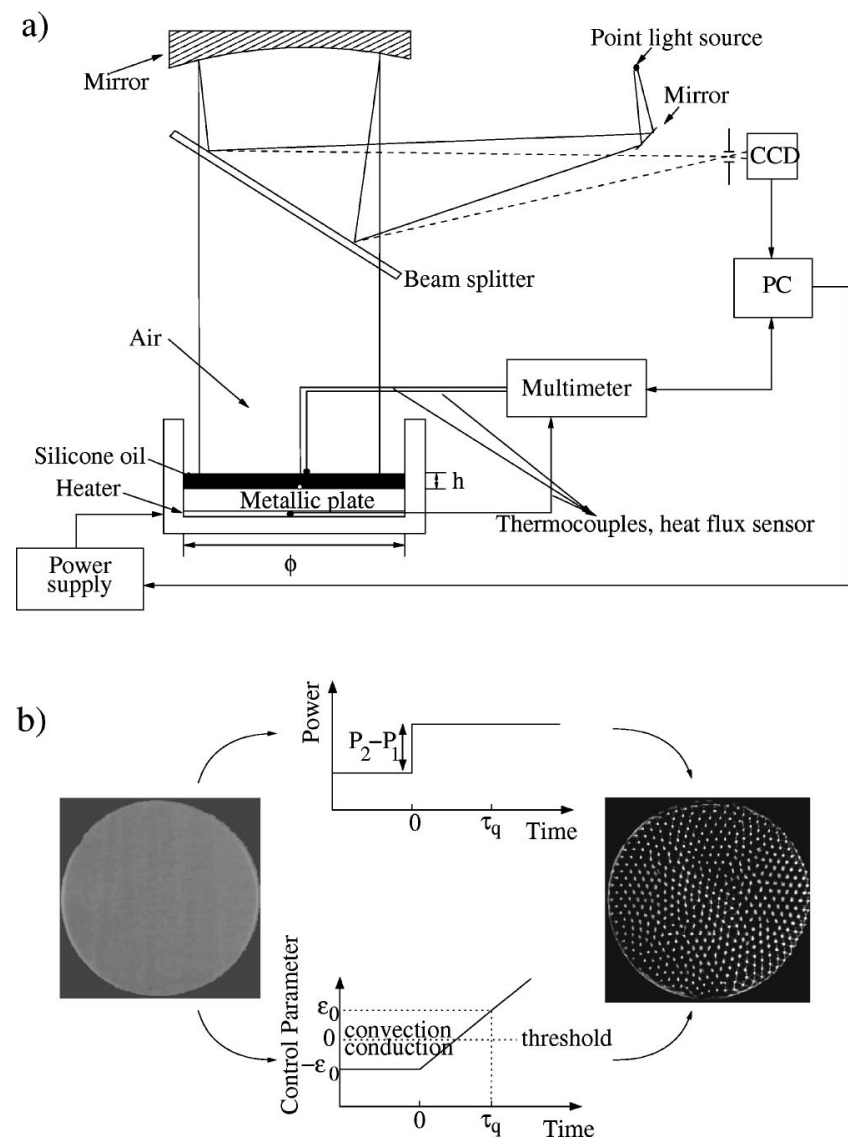

FIG. 1. (a) The experimental setup. (b) Sketch of the experimental process. Starting with a conductive state (left pattern) and applying a step in the delivered power (upper plot), a linear increase in the control parameter is induced (lower plot), leading the system to cross the conduction-convection threshold and to reach a fixed control parameter value after a time $\tau_{q}$. At this moment, a frame (like the one shown in the right pattern) is captured in which the bright points correspond to the hotter fluid going upward.

tivities of the order of the silicone oil). The bottom of the cell is a metallic plate polished on the upper side, and with a glued circular heater on the lower side connected to a computer controlled power supply.

The choice of fluids used in the experiment was dictated mainly by their physical properties, i.e., namely by the facts that they behave as an Oberbeck-Boussinesq fluid for the range of temperatures applied along the whole experimental trial, they are transparent to the light, and they are allowable over a wide range of kinematical viscosities. In particular, the high Prandtl number of the three fluids points out that the velocity field is slaved to the temperature field. Moreover, the control parameter distance between the primary and secondary bifurcations is much larger than the changes performed during the experimental procedure. The fluid layer depths used induce a mostly surface tension driven instability. The relevant physical parameters used in the experiments are reported below [first, second, and third numbers refer to fluid with a kinematic viscosity $\nu$ of 50,100 , and $350 \mathrm{cS}$ (centistokes), respectively]: (a) thermal conductivities $0.1505,0.1557$, and $0.16 \mathrm{~W} \mathrm{~m}^{-1}{ }^{\circ} \mathrm{C}^{-1}$; (b) thermal diffusiv- ity $\kappa=1.037 \times 10^{-7}, 1.067 \times 10^{-7}$, and $1.13 \times 10^{-7} \mathrm{~m}^{2} \mathrm{~s}^{-1}$; (c) Prandtl numbers: 482, 937 and $3097(\nu / \kappa)$; (d) fluid depths 1.3, 1.4, and $1.9 \mathrm{~mm}$; (e) and diameters of the cell 127, 137, and $137 \mathrm{~mm}$.

Local temperatures were measured by three T-type thermocouples [see Fig. 1(a)], the first one located under the metallic plate $\left(T_{1}\right)$, the second over the metallic plate $\left(T_{2}\right)$, and the third on the fluid surface $\left(T_{3}\right) . T_{2}$ and $T_{3}$ were put in the cell center only when necessary for the measurement. All thermocouples are connected with a computer controlled multimeter. Information on the global temperature field is gathered through shadowgraph-type techniques [13], and the resulting image is captured by a charge-coupled-device camera and sent to a computer.

Let us summarize the measurement trial. The system is initially set in a stationary conductive state [like the one shown in Fig. 1(b), left] just below the convective threshold by applying a power $P_{1}$ to the heater. The control parameter is the reduced temperature difference, defined by $\epsilon=(\Delta T$ $\left.-\Delta T_{c}\right) / \Delta T_{c}$, where $\Delta T=T_{2}-T_{3}$, and $\Delta T_{c}$ is the $\Delta T$ in which the conduction state is linearly unstable. Then, we suddenly increase the power delivered to the system to $P_{2}$ [see the curve in Fig. 1(b), top]. As a consequence, $\epsilon$ increases linearly [see the curve in Fig. 1(b), bottom], with a slope depending on $P_{2}-P_{1}$. The linear dependence of $\epsilon$ vs $P_{2}-P_{1}$ was verified by a previous learning section. For each chosen viscosity, a well defined temperature $T_{0}$ exists which corresponds to a formed pattern with a value of the control parameter equal to $+\epsilon_{0}$, which depends on the measurement. We furthermore define the quench time $\tau_{q}$, the time that $T_{1}$ requires to attain a $T_{0}$ value, assuming the initial time to be the instant at which the linear ramp of the control parameter begins. After $\tau_{q}$, an image of the pattern is captured. This process is repeated ten times for each slope $\partial \epsilon / \partial t$, this number being a satisfactory compromise between the reduction of the statistic error and the time spent in each measurement (of the order of hours).

This way one obtains images [like the one shown in Fig. 1(b), right] where the bright zones correspond to hot points with the fluid going upwards. The image analysis begins with a program determining the hot point positions, and building its corresponding Voronoi cell [14], thus identifying the connectivity properties of each point (coordination number, etc.). Since the structure emerging in a BénardMarangoni convection near the primary bifurcation is a hexagonal pattern, the centers of the hexagons form a collection of points, each one having six nearest neighbors. The most stable topological defect is the penta-hepta structure, leading to two adjacent points with five and seven nearest neighbors each. On the other side, in a state far from stationarity many other defects may be observed, thus making complicated the use of complex demodulation techniques. As a consequence, our approach to count defects consists of considering them as one of the following possibilities [15]: (a) points with a coordination number different from 6, (b) points with a coordination number equal to 5 , and (c) points with a coordination number equal to 7 (mostly corresponding to penta-hepta defects). 
It is important to remark that, in general, all these counting methods introduce a coarsening spatial scale of the order of the pattern wavelength. However, this problem becomes relevant only for defect densities greater than (or of the order of) 1 , which is not the case presented in this paper.

Let us report how the density of defects $[\rho$ $=($ number of defects $) /($ number of hot points $)]$ depends upon $\tau_{q}$. The aspect ratio $a \equiv \phi / \lambda$ is the ratio between the diameter of the cell $\phi$ and the characteristic wavelength of the pattern $\lambda$. In our experiment $a$ is 25,22 , and 15 for 50, 100 , and $350 \mathrm{cS}$, respectively.

Several time scales in our experiment contribute to limiting the range for the choice of $\tau_{q}$. In fact, in order to make comparisons with the relevant time scales associated with the pattern formation, it is useful to refer to $\tau_{q}^{\prime}=\tau_{q} / 2$, which marks the time interval the system is above threshold. The first time scale is imposed by our power supply and the thermal inertia of the metallic plate (of the order of $80 \mathrm{~s}$ ), limiting the hand of fast quenches. A further limit to small $\tau_{q}^{\prime}$ comes from the vertical thermal diffusion time, defined as the ratio of the square of the fluid depth to the thermal diffusivity (from the parameters reported above, this may be estimated as 16, 18, and $32 \mathrm{~s}$, for viscosities of 50, 100, and $350 \mathrm{cS}$ respectively). A natural limit to large $\tau_{q}^{\prime}$ comes from the following. Due to the particular boundary conditions imposed in our setup, the resulting temperature gradients are spatially nonuniform, the dissipated energy being larger at the periphery than at the center of the heater. As a consequence, a drift process of the structure from the center to the periphery is induced, making the center a source of defects, and the boundary a defect sink. In other words, during the formation of the cellular pattern, defects are nucleated close to the cell center, advected by the drift process toward the cell boundaries, and eventually annihilated in the cell boundary layer. In order for our statistics not to be altered by the above process, we limit the calculation of the defect density in the central area of the pattern (that is, we do not count defects whose distance is less than $2 \lambda$ from the boundaries, this way also avoiding accounting for all spurious defects emerging within the boundary layer area), as well as we limit $\tau_{q}^{\prime}$ to be smaller than the drift characteristic time $t_{h}$ $=\left(\lambda / v_{\text {hexagons }}\right) \sim 2304,2616$, and $3840 \mathrm{~s}$ for viscosities of 50,100 , and $350 \mathrm{cS}$ respectively ( $v_{\text {hexagons }}$ being the measured drift velocity of the structure).

Furthermore, once $\tau_{q}^{\prime}$ has been selected, one has to take care that the quench time is compatible with the mean annihilation time of defects $t_{d}$, to prevent the defect statistic from being altered by defect annihilation processes, whose time scale can be estimated as the ratio of the mean defectdefect distance $l$ to the mean defect velocity $v_{d}$. By assuming $l$ to be equal the correlation length (which depends upon $\tau_{q}^{\prime}$ ) and by estimating $v_{d}$, the associated $t_{d}=l / v_{d}$ comes out to be of the order of 2850, 2500, and 6000 s when the largest $\tau_{q}^{\prime}$ is considered in the measurements for viscosities of 50, 100, and $350 \mathrm{cS}$, respectively. As a result of all the above limitations, the smaller (larger) $\tau_{q}^{\prime}$ used in the experiment are 82 (1550), 96 (2045), and 103 (2182)s for 50, 100, and $350 \mathrm{cS}$, respectively.
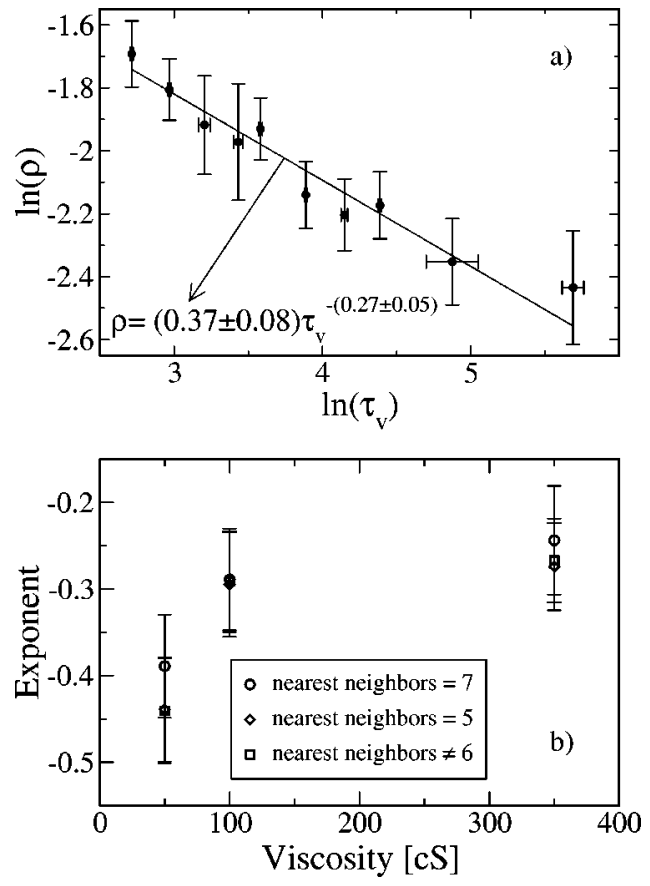

FIG. 2. (a) Defect density (adimensional quantity) vs $\tau_{v}$ (adimensional quantity) for a viscosity of $350 \mathrm{cS}$. Both horizontal and vertical axes are on a logarithmic scale. The defect counting method corresponds to a coordination number of 5. (b) Scaling exponent for the defect density (vertical axis, adimensional quantity) vs the viscosity of the fluid (horizontal axis, in cS). Results are drawn for the three counting methods, giving the same result. The legend in the inset specifies the particular method used to measure the different points represented in the plot.

In all cases the number of measured defects is much larger than 50, usually being around 75. This fact warrants that we have enough statistics to extract results about scaling law properties.

From an experimental point of view, $\tau_{q}$ depends on the initial and final chosen values of $\Delta T$. However, this dependence can be avoided by taking new measurements of the temperature differences $\Delta T$ in the fluid during the power ramp delivery. This way, one obtains the slope of the temperature difference vs time $1 / \tau_{v}=\left[\Delta T\left(t=\tau_{q}\right)-\Delta T(t\right.$ $=0)] / \tau_{q}$. Now $\tau_{v}$ plays the same role as the quench time, but it does not depend on the initial and final points. Four measurements of this type for each ramp have been performed. With the help of 40 temperature measurements corresponding to ten different ramps, a function to transform $\tau_{q}$ in $\tau_{v}$ is obtained.

In Fig. 2(a) we report the defect density versus $\tau_{v}$. Each curve point is the mean of ten measurements, and the error bars in the two axes are the standard deviations of the density of defects and of $\tau_{v}$. The best fit for this curve is a power law. Similar curves are obtained for the other counting methods, and also for the other chosen viscosities.

Figure 2(b) reports the power law exponent for the three viscosities, with their errors. Within the experimental error, the results are the same for all the counting methods used, and point to an exponent always greater than $-\frac{1}{2}$. Only the method that considers defects as points with coordination 
numbers equal to 7 differs from the other two methods. One explanation for this fact is the existence of defects formed only by points with a coordination number equal to 5 . Consequently, this kind of defect is not taken into account by this method.

In conclusion, our results show that the density of defects vs the characteristic quench time follows a scaling law for a conduction-convection transition in a Bénard-Marangoni system. The values of the scaling exponents increase as the viscosity increases, for the whole range of considered viscosities.

The values for the exponents differ from the ones predicted by Zurek for condensed matter systems. A possible explanation for this difference may rely on the fact that our experiment displays defects which do not result from zeros of the global field amplitude, and the dynamics of the three different modal amplitudes concurring to the formation of the structure should be taken into account. Another possible reason could be related to the fact that our case cannot be encompassed within the assumption of mean field theory [16].

The authors acknowledge F. T. Arecchi, M. Bestehorn, J. Burguete, J. Kurths, D. Maza, I. Procaccia, and P. L. Ramazza for useful discussions. Work was partly supported by Italy-Spain integrated action HI97-30 and by the Spanish DGICYT Contract No. PB98-0208. S. C. acknowledges financial support from the "Asociación de Amigos de la Universidad de Navarra.' S. B. acknowledges financial support from EU Contract No. ERBFMBICT983466.
[1] M.C. Cross and P.C. Hohenberg, Rev. Mod. Phys. 65, 851 (1993).

[2] M. Berry, in Physics of Defects, edited by R. Balian, M. Kléman, and J. P. Poirier (North-Holland, Amsterdam, 1981), pp. 456-543; N.B. Baranova and B. Ya Zel'dovich, Zh. Éksp. Teor. Fiz. 80, 1789 (1981) [Sov. Phys. JETP 53, 925 (1981)]; P. Coullet, C. Elphick, L. Gil, and J. Lega, Phys. Rev. Lett. 59, 884 (1987).

[3] F.T. Arecchi, G. Giacomelli, P.L. Ramazza, and S. Residori, Phys. Rev. Lett. 67, 3749 (1991).

[4] G. Ahlers and R.P. Behringer, Suppl. Prog. Theor. Phys. 64, 186 (1979).

[5] S. Ciliberto, P. Coullet, J. Lega, E. Pampaloni, and C. PérezGarcia, Phys. Rev. Lett. 65, 2370 (1990).

[6] J. P. Gollub and R. Ramshankar, in New Perspective in Turbulence, edited by S. Orszag and L. Sidorovich (Springer, Berlin, 1990).

[7] K. Kawasaki, Prog. Theor. Phys. Suppl. 79, 161 (1984); E. Bodenschatz, W. Pesch, and L. Kramer, Physica D 32, 135 (1988)

[8] T.W.B. Kibble, J. Phys. A 9, 1387 (1976); W.H. Zurek, Phys.
Rep. 276, 177 (1996)

[9] P.C. Hendry, N.S. Lawson, R.A.M. Lee, P.V.E. McClintock, and C.D.H. Williams, Nature (London) 368, 315 (1994); V.M.H. Ruutu, V.B. Eltsov, A.J. Gill, T.W.B. Kibble, M. Krusius, Y.G. Makhlin, B. Placais, G.E. Volovik, and Wen Xu, ibid. 382, 334 (1996); C. Bäuerle, Y.M. Bunkov, S.N. Fischer, H. Godfron, and G.R. Pickett, ibid. 382, 332 (1996).

[10] I. Chuang, R. Dürrer, N. Turok, and B. Yurke, Science 251, 133 (1991); M.J. Bowick, L. Chandar, E.A. Schiff, and A.M. Srivastava, ibid. 263, 943 (1994).

[11] S. Ducci, P.L. Ramazza, W. González-Viñas, and F.T. Arecchi, Phys. Rev. Lett. 83, 5210 (1999).

[12] M.F. Schatz, S.J. VanHook, W.D. McCormick, J.B. Swift, and H.L. Swinney Phys. Rev. Lett. 75, 1938 (1995).

[13] H. Mancini, Ph.D thesis, Universidad de Navarra, 1994.

[14] G. Voronoi, J. Reine Angew. Math. 134, 198 (1908).

[15] P. Cerisier, S. Rahal, and B. Billia, Phys. Rev. E 54, 3508 (1996).

[16] R. K. Pathria, Statistical Mechanics (Butterworth-Heinemann, Oxford, 1996), p. 336. 